\title{
Structure of Groups with Generalized Normal Subgroups
}

\author{
Mohammad Arshaduzzaman \\ Department of Mathematics, College of Science, Al Baha University, Al Baha, KSA \\ Email: shayan.zaman@gmail.com
}

Received 14 April 2014; revised 14 May 2014; accepted 21 May 2014

Copyright (C) 2014 by author and Scientific Research Publishing Inc.

This work is licensed under the Creative Commons Attribution International License (CC BY). http://creativecommons.org/licenses/by/4.0/

c) (i) Open Access

\section{Abstract \\ The present paper deals with a subgroup $X$ of a group $G$ is almost normal if the index $\mid G$ : $N G(X) \mid$ is finite, while $X$ is nearly normal if it has finite index in the normal closure $X G$. This paper investi- gates the structure of groups in which every (infinite) subgroup is either almost normal or nearly normal.}

\section{Keywords}

\section{Normality, Nearly Normality, Normalizer Subgroup}

\section{Introduction}

A subgroup $X$ of a group $G$ is said to be almost normal if it has only finitely many conjugates, or equivalently if the normalizer $N G(X)$ of $X$ has finite index in $G$. A famous result of B. H. Neumann [1] states that all subgroups of a group $G$ are almost normal if and only if the centre $Z(G)$ has finite index in $G$. This theorem was later generalized by I. I. Eremin [2], who showed that it is enough to impose the almost normality restriction just to abelian subgroups. Another relevant type of generalized normality is the so-called nearly normality: a subgroup $X$ of a group $G$ is nearly normal if it has finite index in its normal closure $X G$. In the above quoted paper, Neumann also proved that all subgroups of a group $\mathrm{G}$ are nearly normal if and only if the commutator subgroup $G^{0}$ of $G$ is finite; also in this case, it is enough to suppose that all abelian subgroups are nearly normal (a result that was obtained by M. J. Tomkinson [3]). The concepts of almost normal and nearly normal subgroups are in general incomparable. For instance, all subgroups of the base group of the standard wreath product $W=Z_{0} Z^{2}$ are almost normal in W, but only few of them are nearly normal; moreover, if $G$ is any group containing an infinite minimal normal subgroup $N$, which is abelian of prime exponent, then each proper subgroup of finite index of $N$ is nearly normal in $G$ but has infinitely many conjugates. On the other hand, it follows from Neumann's results and from the celebrated theorem of Schur on the finiteness of the commutator subgroup of central-by-finite 
groups (see for instance [4] Part 1, Theorem 4.12) that if all subgroups of a group $G$ are almost normal, then they must be also nearly normal. It should also be mentioned here that the structure of groups in which every infinite subgroup is almost normal and that of groups in which all infinite subgroups are nearly normal have been described in [5] and [6], respectively; it turns out that the information that all subgroups of a certain type are almost normal (or that they all are nearly normal) is of some relevance. The aim of this paper is to study groups in which every member of a relevant system of subgroups is either almost normal or nearly normal; in particular, Tomkinson's theorem quoted above will be generalized to this situation.

A result of Y. D. Polovicki 1 [7] shows that a group $G$ has finitely many normalizers of abelian subgroups if and only if it is finite over the centre. In recent years, many other papers have appeared on the structure of groups with finitely many normalizers of subgroups with a given property (see for instance [8]-[12]); here we will also consider groups with finitely many normalizers of subgroups which are neither almost normal nor nearly normal.

Most of our notation is standard and can be found in [4].

\section{Abelian Subgroups}

Recall that the F C-centre of a group $G$ is the subgroup consisting of all elements with finitely many conjugates, and $G$ is an F C-group if it coincides with its F C-centre. The theory of F C-groups plays an important role in many questions concerning infinite groups with finiteness conditions; we refer to the monograph [13] for the main properties of F C-groups.

It is easy to show that a cyclic subgroup $h x i$ of a group $G$ is almost normal if and only if it is nearly normal, and both such properties are also equivalent to the fact that the conjugacy class of $\mathrm{x}$ in $G$ is finite. Thus a group $G$ is an F C-group if and only if all its cyclic subgroups are almost normal (or nearly normal). It turns out that, within the universe of F C-groups, almost normality is a stronger property than nearly normality.

Lemma 2.1. Let $G$ be an F C-group, and let $X$ be an almost normal subgroup of $G$. Then $X$ is nearly normal in G.

Proof: As the normalizer $N G(X)$ has finite index in $G$, there exists a finitely generated subgroup $E$ of $G$ such that $G=h E, N G(X) i$. Moreover, the subgroup $E$ can be chosen normal in $G$, since its normal closure $E G$ is likewise finitely generated. Thus $G=N G(X) E$ and so $X G=X E$. Clearly, $E$ is central-by-finite, so that it satisfies the maximal condition on subgroups, and in particular its subgroup $[X, E]$ is finitely generated. On the other hand, the commutator subgroup $G^{0}$ of $G$ is locally finite, and hence $[X, E]$ is finite. Therefore the subgroup $X$ has finite index in its normal closure $X G=X[X, E]$, and so it is nearly normal in $G$.

The main result of this section is an extension of Tomkinson's theorem quoted in the introduction to the case in which every (abelian) subgroup is either almost normal or nearly normal; it will be obtained as a consequence of a theorem on groups with finitely many normalizers of subgroups with a suitable property. For such purpose, we need a result of B. H. Neumann [14], who holds in the more general situation of groups covered by cosets of subgroups.

Lemma 2.2. Let the group $G=X_{1} \cup \cdots \cup X_{t}$ be the union of finitely many subgroups $X_{1}, \cdots, X_{t}$. Then all $X_{i}$ 's of infinite index can be omitted from this decomposition; in particular, at least one of the subgroups $X_{1}, \cdots, X_{t}$ has finite index in $G$.

Theorem 2.3. Let $G$ be a group with finitely many normalizers of abelian subgroups which are neither almost normal nor nearly normal. Then the commutator subgroup $G^{0}$ of $G$ is finite, and so all subgroups of $G$ are nearly normal.

Proof: Let

$$
\left\{N_{G}\left(X_{1}\right), \cdots, N_{G}\left(X_{k}\right)\right\}
$$

be the set of all normalizers of abelian subgroups of $G$ which are neither almost normal nor nearly normal. If $x$ is any element of $G$ having infinitely many conjugates, we have that $N_{G}(h x i)=N_{G}\left(X_{j}\right)$ for some $j \leq k$, so that in particular $\mathrm{x}$ belongs to $N_{G}\left(X_{j}\right)$ and hence

$$
G=F \cup N_{G}\left(X_{1}\right) \cap N_{G}\left(X_{k}\right)
$$

where $F$ is the F C-centre of $G$. On the other hand, each normalizer $N G(X i)$ has infinite index in $G$, and so it follows from Lemma 2.2 that $G=F$ is an F C-group. The same result shows that the set 


$$
N_{G}\left(X_{1}\right) \cup \cdots \cup N_{G}\left(X_{k}\right)
$$

is properly contained in $G$, so that we may consider an element $g$ of

$$
G W\left(N_{G} X_{1}\right) \cup\left(N_{G}\left(X_{k}\right)\right)
$$

Clearly, $g$ normalizes all subgroups of its centralizer $C G(g)$, so that each abelian subgroup of $C G(g)$ must be either almost normal or nearly normal. Therefore all abelian subgroups of $C G(g)$ are nearly normal by Lemma 2.1, and hence $C G(g)$ has finite commutator subgroup by Tomkinson's theorem. As the index $\mid G$ : $C G(g) \mid$ is finite, the group $G$ is finite-by-abelian-by-finite. On the other hand, any abelian-by-finite F C-group is central-by-finite, and so it follows from Schur's theorem that the commutator subgroup $G^{0}$ of $G$ is finite. In particular, all subgroups of $\mathrm{G}$ are nearly normal.

Corollary 2.4. Let $G$ be a group in which every abelian subgroup is either almost normal or nearly normal. Then the commutator subgroup $G^{0}$ of $G$ is finite, and so all subgroups of $G$ are nearly normal.

\section{Infinite Subgroups of Locally Finite Groups}

It was remarked in Section 2 that any almost normal subgroup of an F C-group is nearly normal. Our next lemma shows in particular that a converse conclusion holds in the case of groups with finite Prufer rank.

Lemma 3.1. Let $G$ be a group, and let $X$ be a nearly normal subgroup of $G$. If $X$ has finite Prufer rank, then it is almost normal in $G$.

Proof: Put $|X G: X|=n$. Then the subgroup $(X G) n$ ! is contained in $X$ and hence the factor group $X G / X G$ has finite exponent. Moreover, $X G / X G$ obviously is residually finite and has finite Prufer rank, so that a relevant result by Mann and Segal (see [15], Theorem A) can be applied to show that $X G / X G$ is locally finite. On the other hand, any abelian subgroup of $X G / X G$ is finite, and hence it follows from the Hall-Kulatilaka-Kargapolov theorem that $X G / X G$ itself is finite (see for instance [4] Part 1, Theorem 3.43). Therefore $X$ is almost normal in $G$.

In order to study groups with finitely many normalizers of infinite subgroups which are neither almost normal nor nearly normal we need a series of lemmas.

Lemma 3.2. Let $G$ be a group, and let $A$ be an infinite abelian normal subgroup of finite index of $G$. If $A$ is the direct product of a collection of subgroups of prime order, then there exists a sequence (an) $n \in N$ of elements of $A$ such that

$$
h a_{n} \mid n \in N i^{G}=\underset{n \in N}{D r} h a_{n} i_{G}
$$

Proof: Let $a_{1}$ be any non-trivial element of $A$, and suppose by induction that elements $a_{1}, \cdots, a_{n}$ of $A$ have been chosen in such a way that

$$
h a_{1}, \cdots, a_{n} i_{G} X \cdots X h a_{n} i_{G}
$$

Clearly, the subgroup $h a_{1}, \cdots, a_{n} i^{G}$ is finite, and hence A contains an infinite subgroup $B$ such that

$$
A=h a_{1}, \cdots, a_{n} i^{G} X B
$$

Since $B$ has finitely many conjugates in $G$, its core $B G$ has finite index in $A$ and so it is infinite. If $a n+1$ is any non-trivial element of $B G$, we have

$$
h a_{1}, \cdots, a_{n}, a_{n+1} i^{G}=h a_{1} i^{G} X \cdots X h a_{n} i^{G} X h a_{n+1} i^{G}
$$

and the lemma is proved.

Lemma 3.3. Let $G$ be a locally finite group, and let $g$ be an element of $G$ such that $X g=X$ for each infinite subgroup $X$ of $G$ which is neither almost normal nor nearly normal. If $g$ has infinitely many conjugates, then all abelian $h g i$-invariant subgroups of $G$ satisfy the minimal condition.

Proof: Assume for a contradiction that there exists an abelian $h g i$-invariant subgroup $A$ of $G$ which does not satisfy the minimal condition. Then the socle of $A$ is infinite, and so by Lemma 3.2 there exist infinite hgi-invariant subgroups $A 1$ and $A 2$ of $A$ such that

$$
\begin{gathered}
A_{1} \cap A_{2}=h A_{2}, A_{2} i \cap h g i=\{1\} \\
h g, A_{1} i \cap h g, A_{2} i=h g i
\end{gathered}
$$


Moreover, by hypothesis each of the infinite subgroups $h g, A_{1} i$ and $h g, A_{2} i$ is either almost normal or nearly normal, but they cannot have the same generalized normality property. Without loss of generality, we suppose that $h g, A_{1} i$ is almost normal and $h g, A_{2} i$ is nearly normal in $G$. Then the normalizer $L=N_{G}\left(h g, A_{1} i\right)$ has finite index in $G$, and so there exists a finite subgroup $E$ of $G$ such that $G=L E$. On the other hand, the subgroup $h g$, $A_{2} i \cap L$ is nearly normal in $L$, so that

$$
h g i=h g, A_{1} i \cap\left(h g, A_{2} i \cap L\right)
$$

is likewise nearly normal in $L$, and hence the subgroup $h g i^{L}$ is finite. Thus also the normal closure

$$
h g i^{G}=h g i^{L E}=h h g i^{L}, E i
$$

is finite. This contradiction proves the lemma.

Corollary 3.4. Let $G$ be a locally finite group, and let $g$ be an element of $G$ such that $X g=X$ for each infinite subgroup $X$ of $G$ which is neither almost normal nor nearly normal. If $g$ has infinitely many conjugates, then its centralizer $C G(g)$ is a Cernikov group.

Proof: It follows from Lemma 3.3 that all abelian subgroups of the centralizer $C G(g)$ satisfy the minimal condition. Therefore $C G(g)$ satisfies the minimal condition on abelian subgroups, and hence it is a Cernikov group by a relevant result of Sunkov [16].

In order to prove that locally finite groups in our situations are close to be locally soluble, we need the following result of B. Hartley [17] on locally finite groups admitting an automorphism of prime-power order for which the set of fixed points is small.

Lemma 3.5. Let $G$ be a locally finite group admitting an automorphism $\alpha$ of prime-power order such that the set of all elements of $G$ fixed by $\alpha$ is a Cernikov group. Then $G$ contains a locally soluble subgroup of finite index.

Lemma 3.6. Let $G$ be a locally finite group with finitely many normalizers of infinite subgroups which are neither almost normal nor nearly normal. Then $G$ contains a locally soluble subgroup of finite index.

Proof: Let $F$ be the F C-centre of $G$, and let

$$
\left\{N_{G}\left(X_{1}\right), \cdots, N_{G}\left(X_{k}\right)\right\}
$$

be the set of all normalizers of infinite subgroups of $G$ which are neither almost normal nor nearly normal. Clearly, each $N_{G}\left(X_{i}\right)$ has infinite index in $G$, and so it follows from Lemma 2.2 that either $G$ is an F C-group or the set

$$
F \cup N_{G}\left(X_{1}\right) \cup \cdots \cup N_{G}\left(X_{k}\right)
$$

is properly contained in $G$. If $G$ is an F C-group, then it has finitely many normalizers of subgroups which are neither almost normal nor nearly normal, and hence $G$ is soluble-by-finite by Theorem 2.3. Suppose now that

$$
F \cup N_{G}\left(X_{1}\right) \cup \cdots \cup N_{G}\left(X_{k}\right)
$$

is a proper subset of $G$, and let $g$ be an element of prime-power order in the set

$$
F \cup N_{G}\left(X_{1}\right) \cup \cdots \cup N_{G}\left(X_{k}\right)
$$

Then $g$ satisfies the condition of Corollary 3.4, so that its centralizer $C G(g)$ is a Cernikov group, and hence $G$ contains a locally soluble subgroup of finite index by Lemma 3.5 .

Lemma 3.7. Let $G$ be a group with finitely many normalizers of infinite subgroups which are neither almost normal nor nearly normal. Then $G$ contains a characteristic subgroup $M$ of finite index such that the normalizer $N M(X)$ is normal in $M$ for each infinite subgroup $X$ of $M$ which is neither almost normal nor nearly normal in $G$.

Proof: If $X$ is any infinite subgroup of $G$ which is neither almost normal nor nearly normal, the normalizer $N G(X)$ has obviously finitely many images under automorphisms of $G$; in particular, the subgroup $N G(X)$ has finitely many conjugates in $G$ and so the index $|G: N G(N G(X))|$ is finite. It follows that also the characteristic subgroup

$$
M(X)=N_{G} \quad\left(N_{G}(X)\right)^{\alpha} \quad \alpha \in \text { Aut } G
$$

has finite index in $G$. Let $\Lambda$ be the set of all infinite subgroups of $G$ which are neither almost normal nor nearly 
normal. If $X$ and $Y$ are elements of $\Lambda$ such that $N G(X)=N G(Y)$, then $M(X)=M(Y)$, and hence also

$$
M=m(X) \quad X \in A
$$

is a characteristic subgroup of finite index of $G$. Let $X$ be any infinite subgroup of $M$ which is neither almost normal nor nearly normal. Then

$$
M \leq M(X) \leq N_{G}\left(N_{G}(X)\right)
$$

and so the normalizer $N M(X)=N G(X) \cap M$ is a normal subgroup of $M$.

Finally, we mention the following result of D. I. Zaicev [18], which is needed in our proofs.

Lemma 3.8. Let $G$ be a locally soluble group, and let $\Gamma$ be a finite group of automorphisms of $G$. If all abelian $\Gamma$-invariant subgroups of $G$ satisfy the minimal condition, then $G$ is a Cernikov group.

We are now in a position to prove the main result of this section. Of course, it describes in particular the structure of locally finite groups in which every infinite subgroup is either almost normal or nearly normal.

Theorem 3.9. Let $G$ be a locally finite group with finitely many normalizers of infinite subgroups which are neither almost normal nor nearly normal. Then either the commutator subgroup $G^{0}$ of $G$ is finite or $G$ is a Cernikov group whose infinite subgroups are almost normal.

Proof: Suppose first that $G$ is an F C-group. Then $G$ has finitely many normalizers of subgroups which are neither almost normal nor nearly normal, and hence the commutator subgroup $G^{0}$ of $G$ is finite by Theorem 2.3.

Assume now that the F C-centre F of $G$ is a proper subgroup, and let

$$
\left\{N_{G}\left(X_{1}\right), \cdots, N_{G}\left(X_{k}\right)\right\}
$$

be the set of all normalizers of infinite subgroups of $G$ which are neither almost normal nor nearly normal. It follows from Lemma 2.2 that the subset

$$
F \cup N_{G}\left(X_{1}\right) \cup \cdots \cup N_{G}\left(X_{k}\right)
$$

is properly contained in $G$, and so we may consider an element $g$ in the set

$$
G W F \cup N_{G}\left(X_{1}\right) \cup \cdots \cup N_{G}\left(X_{k}\right)
$$

Clearly, $g$ satisfies the conditions of Lemma 3.3, and hence all abelian hgi-invariant subgroups of $G$ satisfy the minimal condition. As $G$ contains a locally soluble (normal) subgroup of finite index by Lemma 3.6, it follows from Lemma 3.8 that $G$ is a

Cernikov group. Finally, in this case all nearly normal subgroups of $G$ are almost normal by Lemma 3.1, and hence $G$ has only finitely many normalizers of infinite subgroups which are not almost normal. Let $X$ be any infinite subgroup of $G$, and let $J$ be the finite residual of $X$. Then $J$ is infinite, and so $N G(J) / J$ has only finitely many normalizers of infinite index; another application of Lemma 2.2 yields now that $N G(J) / J$ is an F C-group, so that it is central-by-finite. Thus $X$ is almost normal in $N G(J)$ and so even in $G$, as the index $|G: N G(J)|$ is finite. Therefore all infinite subgroups of $G$ are almost normal.

\section{Main Results: Now We Prove Our Main Results}

\section{Infinite Subgroups of Non-Periodic Groups}

As we mentioned in the introduction, the structure of groups in which all infinite subgroups are almost normal has been described by L. A. Kurdachenko, S. S. Levishchenko and N. N. Semko [5], while that of groups in which all infinite subgroups are nearly normal has been studied by S. Franciosi and F. de Giovanni [6]. We state here as lemmas parts of their results.

Lemma 4.1. Let $G$ be a non-periodic group whose infinite subgroups are almost normal. Then either $G$ is central-by-finite or it contains normal subgroups $N$ and $A$ such that $N \leq A, N$ is finite, G/A has prime order $p$ and $A / N$ is a free abelian group of rank $p-1$ on which $G / A$ acts rationally irreducibly.

Lemma 4.2. Let $G$ be a non-periodic group whose infinite subgroups are nearly normal. Then either $G^{0}$ is finite or $G$ is a finite extension of an infinite cyclic subgroup.

It was observed in the introduction that if all subgroups of a group $G$ are almost normal, then each of them must be nearly normal. The following example shows that the imposition of almost normality just to infinite 
subgroups does not have similar consequences. Let the group

$$
G=h x i n(h a i X h b i)
$$

be the semidirect product of a free abelian group of rank 2 by a cyclic group $h x i$ of order 3 , acting by the rules $a x$ $=b$ and $b x=a^{-1} b^{-1}$. Then all infinite subgroups of $G$ are almost normal, but hai has obviously infinite index in its normal closure. On the other hand, Lemma 4.2 shows that if $G$ is a non-periodic group in which every infinite subgroup is nearly normal, then either $G^{0}$ is finite (and so all subgroups are nearly normal) or all infinite subgroups of $G$ are almost normal. Our next result proves that the same conclusion holds in a more general situation.

Theorem 4.3. Let $G$ be a non-periodic group in which every infinite subgroup is either almost normal or nearly normal. Then either the commutator subgroup $G^{0}$ of $G$ is finite or all infinite subgroups of $G$ are almost normal.

Proof: Let $a$ be any element of infinite order of $G$. Then the cyclic subgroup hai is either almost normal or nearly normal, and hence the conjugacy class of $a$ is finite. In particular, the normal closure hai $G$ is a finitely generated F C-group, and so it is central-by-finite. Moreover, every subgroup of G/haiG is either almost normal or nearly normal, so that the commutator subgroup of $G /$ haiG is finite by Corollary 2.4 and hence $G$ is soluble-by-finite.

Let $T$ be the largest periodic normal subgroup of $G$, and assume first that $T$ is infinite. Then each subgroup of $G / T$ is either almost normal or nearly normal, and hence the commutator subgroup of $G / T$ is finite by Corollary 2.4. It follows that the commutator subgroup of $G$ is periodic, so that $T$ is the set of all elements of finite order of $G$ and $G$ is generated by its elements of infinite order. Thus $G$ is an F C-group by the first part of the proof, and so each subgroup of $G$ is either almost normal or nearly normal. Again Corollary 2.4 shows that in this case all subgroups of $G$ are nearly normal, and hence $G^{0}$ is finite.

Suppose now that $T$ is finite. If all infinite subgroups of $G / T$ are nearly normal, we have of course that also all infinite subgroups of $G$ are nearly normal; on the other hand, if all infinite subgroups of $G / T$ are almost normal, it follows from Lemma 4.1 that either the commutator subgroup of $G / T$ is finite (and in this case $G^{0}$ is finite, and so again all infinite subgroups of $G$ are nearly normal) or $G$ has finite Prufer rank, and so by Lemma 3.1 all infinite subgroups of $G$ are almost normal. Therefore it is enough to prove that either all infinite subgroups of $G / T$ are almost normal or all infinite subgroups of $G / T$ are nearly normal, and hence by replacing $G$ by the factor group $G / T$, it can be assumed without loss of generality that $G$ has no periodic non-trivial normal subgroups. In particular, the Hirsch-Plotkin radical $H$ of $G$ is torsion-free, so that each element of $H$ has finitely many conjugates and hence $H$ is abelian. If $h$ is any non-trivial element of $H$, the index $\mid h h i G$ : hhi| is finite, so that $h h i G$ is infinite cyclic and then $h$ hi is normal in $G$. Therefore all cyclic subgroups of $H$ are normal in $G$, and $G / C G(H)$ is isomorphic to a group of power automorphisms of $\mathrm{H}$; in particular, $G / C G(H)$ is finite, as any torsion-free abelian group has precisely two power automorphisms. Let $S$ be the largest soluble normal subgroup of $G$. Then $H$ is contained in $S$ and $C S(H)=H$, so that $S / H$ is finite and the factor group $G / H$ is likewise finite. Let $X$ be any infinite subgroup of $G$. Then $X \cap H$ is a non-trivial normal subgroup of $G$, and each subgroup of $G / X \cap H$ is either almost normal or nearly normal. Application of Corollary 2.4 yields that all subgroups of $G / X \cap H$ are nearly normal, so that $X$ is a nearly normal subgroup of $G$. Therefore all infinite subgroups of $G$ are nearly normal in this case, and the theorem is proved.

Our last results provide informations on non-periodic groups with finitely many normalizers of infinite subgroups which are neither almost normal nor nearly normal.

Theorem 4.4. Let $G$ be a non-periodic group in which the elements of finite order form a subgroup. If $G$ has finitely many normalizers of infinite subgroups which are neither almost normal nor nearly normal, then either all infinite subgroups of $G$ are almost normal or all infinite subgroups of $G$ are nearly normal.

Proof: Let $T$ be the subgroup consisting of all elements of finite order of $G$, and consider the $F$ C-centre $F$ of $G$. Assume for a contradiction that the statement is false, so that by Theorem 4.3 the group $G$ must contain some infinite subgroup which is neither almost normal nor nearly normal. Let

$$
\left\{N_{G}\left(X_{1}\right), \cdots, N_{G}\left(X_{k}\right)\right\}
$$

be the set of all normalizers of infinite subgroups of $G$ which are neither almost normal nor nearly normal. Then

$$
G=F \cup T \cup N_{G}\left(X_{1}\right) \cup N_{G}\left(X_{k}\right)
$$


As each of the subgroups $T, N_{G}\left(X_{1}\right) \cdots N_{G}\left(X_{k}\right)$ has infinite index in $G$, it follows from Lemma 2.2 that $G$ $=F$ is an $F$ C-group, and hence all its finite subgroups are almost normal. Therefore $G$ has finitely many normalizers of subgroups which are neither almost normal nor nearly normal, and so the commutator subgroup $G^{0}$ of $G$ is finite by Theorem 2.3. This contradiction proves the theorem.

Corollary 4.5. Let $G$ be a non-periodic group in which the elements of finite order form a subgroup. Then $G$ has finitely many normalizers of infinite subgroups which are neither almost normal nor nearly normal if and only if either $G$ is a finite extension of an infinite cyclic group or the commutator subgroup $G^{0}$ of $G$ is finite.

Proof: Suppose that $G$ has finite many normalizers of infinite subgroups which are neither almost normal nor nearly normal. It follows from Theorem 4.4 that either all infinite subgroups are almost normal or all infinite subgroups of $G$ are nearly normal. In the latter case, either $G^{0}$ is finite or $G$ is a finite extension of an infinite cyclic group by Lemma 4.2. Assume now that all infinite subgroups of $G$ are almost normal. Then it follows from Lemma 4.1 either $G / Z(G)$ is finite or $G$ contains normal subgroups $E$ and $A$ such that $E \leq A, E$ is finite, $G / A$ has prime order $p, A / E$ is free abelian of rank $p-1$ and any element of $G \backslash A$ acts rationally irreducibly on $A / E$. If $G / Z(G)$ is finite, of course we obtain that $G^{0}$ is likewise finite. In the other case, as the elements of finite order in $G$ form a subgroup, we have that $G / E$ must be torsion-free, so that $A / E$ is infinite cyclic and $G$ is a finite extension of an infinite cyclic subgroup. The converse statement is obvious.

Finally, we observe that in our situation the existence of infinite periodic normal subgroups forces the elements of finite order to form a subgroup, and hence allows to apply Theorem 4.4.

Corollary 4.6. Let $G$ be a non-periodic group containing an infinite periodic normal subgroup. If $G$ has finitely many normalizers of infinite subgroups which are neither almost normal nor nearly normal, then the set of all elements of finite order of $G$ is a subgroup.

Proof. Let $N$ be an infinite periodic normal subgroup of $G$. Then the factor group $G / N$ has finitely many normalizers of subgroups which are neither almost normal nor nearly normal, and hence its commutator subgroup is finite by Theorem 2.3. It follows that the commutator subgroup $G^{0}$ of $G$ is periodic, so that the elements of finite order of $\mathrm{G}$ form a subgroup.

\section{Conclusion}

Thus a subgroup $X$ of a group $G$ is almost normal if the index $|G: N G(X)|$ is finite, while $X$ is nearly normal if it has finite index in the normal closure $X G$. This paper investigates the structure of groups in which every (infinite) subgroup is either almost normal or nearly normal.

\section{References}

[1] Neumann, B.H. (1955) Groups with Finite Classes of Conjugate Subgroups. Mathematische Zeitschrift, 63, 76-96. http://dx.doi.org/10.1007/BF01187925

[2] Eremin, I.I. (1959) Groups with Finite Classes of Conjugate Abelian Subgroups. Matematicheskii Sbornik (MatSb) Moscow, 47, 45-54.

[3] Tomkinson, M.J. (1981) On Theorems of B. H. Neumann Concerning F C-Groups. Rocky Mountain Journal of Mathematics, 11, 47-58. http://dx.doi.org/10.1216/RMJ-1981-11-1-47

[4] Robinson, D.J.S. (1972) Finiteness Conditions and Generalized Soluble Groups. Springer, Berlin.

[5] Kurdachenko, L.A., Levishchenko, S.S. and Semko, N.N. (1983) Groups with Almost Normal Infinite Subgroups. Soviet Mathematics (Izvestiya VUZ. Matematika), 27, 73-81.

[6] Franciosi, S. and de Giovanni, F. (1994 \& 1995) Groups Satisfying the Minimal Condition on Certain Non-Normal Subgroups. Proceedings of Groups-Korea, de Gruyter, Berlin, 107-118.

[7] Polovickii, Y.D. (1980) Groups with Finite Classes of Conjugate Infinite Abelian Subgroups. Soviet Mathematics (Izvestiya VUZ. Matematika), 24, 52-59.

[8] De Falco, M., de Giovanni, F. and Musella, C. (2010) Groups with Finitely Many Normalizers of Non-Polycyclic Subgroups. Algebra Colloquium (World Scientific), 17, 203-210. http://dx.doi.org/10.1142/S1005386710000210

[9] De Mari, F. and de Giovanni, F. (2005) Groups with Few Normalizer Subgroups. Irish Mathematical Society Bulletin, 56, 103-113.

[10] De Mari, F. and de Giovanni, F. (2006) Groups with Finitely Many Normalizers of Non-Abelian Subgroups. Ricerche di Matematica, 55, 311-317.

[11] De Mari, F. and de Giovanni, F. (2006) Groups with Finitely Many Normalizers of Subnormal Subgroups. Journal of 
Algebra, 304, 382-396. http://dx.doi.org/10.1016/j.jalgebra.2005.10.006

[12] De Mari, F. and de Giovanni, F. (2007) Groups with Finitely Many Normalizers of Non-Nilpotent Subgroups. Mathematical Proceedings of the Royal Irish Academy. Royal Irish Academy, Dublin, 107A, 143-152. http://dx.doi.org/10.3318/PRIA.2007.107.2.143

[13] Tomkinson, M.J. (1984) FC-Groups, Pitman, Boston.

[14] Neumann, B.H. (1954) Groups Covered by Permutable Subset. Journal of the London Mathematical Society, 29, 236248. http://dx.doi.org/10.1112/jlms/s1-29.2.236

[15] Mann, A. and Segal, D. (1990) Uniform Finiteness Conditions in Residually Finite Groups. Proceedings London Mathematical Society, 61, 529-545.

[16] Sunkov, V.P. (1970) On the Minimality Problem for Locally Finite Groups. Algebra and Logic, 9, 137-151. http://dx.doi.org/10.1007/BF02218982

[17] Hartley, B. (1988) Fixed Points of Automorphisms of Certain Locally Finite Groups and Chevalley Groups. Journal of the London Mathematical Society, 37, 421-436. http://dx.doi.org/10.1112/jlms/s2-37.3.421

[18] Zaicev, D.I. (1974) On Solvable Subgroups of Locally Solvable Groups. Soviet Mathematics Doklady (SSSR), 15, 342345. 